\title{
A narrative review on tumor microenvironment in oligometastatic and oligoprogressive non-small cell lung cancer: a lot remains to be done
}

\author{
Lorenzo Belluomini ${ }^{1} \wedge$, Alessandra Dodi ${ }^{1}$, Alberto Caldart ${ }^{1}$, Dzenete Kadrija ${ }^{1}$, Marco Sposito ${ }^{1}$, \\ Miriam Casali ${ }^{1}$, Giulia Sartori ${ }^{1}$, Miriam Grazia Ferrara ${ }^{2}$, Alice Avancini ${ }^{3}$, Emilio Bria ${ }^{2}$, Jessica Menis ${ }^{4,5}$, \\ Michele Milella $^{1 \# \wedge}$, Sara Pilotto ${ }^{1 \# \wedge}$
}

${ }^{1}$ Section of Oncology, Department of Medicine, University of Verona School of Medicine and Verona University Hospital Trust, Verona, Italy; ${ }^{2}$ Comprehensive Cancer Center, Fondazione Policlinico Universitario Agostino Gemelli IRCCS, Roma, Italy; Department of Translational Medicine and Surgery, Università Cattolica del Sacro Cuore, Roma, Italy; ${ }^{3}$ Biomedical, Clinical and Experimental Sciences, Department of Medicine, University of Verona Hospital Trust, Verona, Italy; ${ }^{4}$ Department of Surgery, Oncology and Gastroenterology, University of Padova, Padova, Italy; ${ }^{5}$ Medical Oncology Department, Istituto Oncologico Veneto IRCCS, Padova, Italy

Contributions: (I) Conception and design: L Belluomini, A Dodi, A Avancini, J Menis, S Pilotto, M Milella; (II) Administrative support: None; (III) Provision of study materials or patients: None; (IV) Collection and assembly of data: L Belluomini, A Dodi, D Kadrija, M Sposito, M Casali, G Sartori; (V) Data analysis and interpretation: L Belluomini, A Dodi, J Menis, S Pilotto, M Milella; (VI) Manuscript writing: All authors; (VII) Final approval of manuscript: All authors.

\#These authors contributed equally for the senior authorship.

Correspondence to: Prof. Michele Milella. Section of Oncology, Department of Medicine, University of Verona School of Medicine and Verona University Hospital Trust, P.le L. A. Scuro 10, 37134 Verona, Italy. Email: michele.milella@univr.it.

\begin{abstract}
Objective: In this review, we aim to collect and discuss available data about the role and composition of tumor microenvironment (TME) in oligometastatic (OMD) and oligoprogressive (OPD) non-small cell lung cancer (NSCLC). Furthermore, we aim to summarize the ongoing clinical trials evaluating as exploratory objective the TME composition, through tissue and/or blood samples, in order to clarify whether TME and its components could explain, at least partially, the oligometastatic/oligoprogressive process and could unravel the existence of predictive and/or prognostic factors for local ablative therapy (LAT).

Background: OMD/OPD NSCLC represent a heterogeneous group of diseases. Several data have shown that TME plays an important role in tumor progression and therefore in treatment response. The crucial role of several types of cells and molecules such as immune cells, cytokines, integrins, protease and adhesion molecules, tumor-associated macrophages (TAMs) and mesenchymal stem cells (MSCs) has been widely established. Due to the peculiar activation of specific pathways and expression of adhesion molecules, metastatic cells seem to show a tropism for specific anatomic sites (the so-called "seed and soil" hypothesis). Based on this theory, metastases appear as a biologically driven process rather than a random release of cancer cells. Although the role and the function of TME at the time of progression in patients with NSCLC treated with tyrosine-kinase inhibitors and immune checkpoint inhibitors (ICIs) have been investigated, limited data about the role and the biological meaning of TME are available in the specific OMD/OPD setting.
\end{abstract}

Methods: Through a comprehensive PubMed and ClinicalTrials.gov search, we identified available and ongoing studies exploring the role of TME in oligometastatic/oligoprogressive NSCLC.

Conclusions: Deepening the knowledge on TME composition and function in OMD/OPD may provide innovative implications in terms of both prognosis and prediction of outcome in particular from local treatments, paving the way for future investigations of personalized approaches in both advanced and early

^ ORCID: Lorenzo Belluomini, 0000-0003-1981-5499; Michele Milella, 0000-0002-3826-5237; Sara Pilotto, 0000-0003-2229-4874. 
disease settings.

Keywords: Tumor microenvironment (TME); non-small cell lung cancer (NSCLC); oligometastases; oligoprogression; local ablative therapy (LAT)

Submitted Oct 20, 2020. Accepted for publication Apr 19, 2021.

doi: $10.21037 /$ tlcr-20-1134

View this article at: http://dx.doi.org/10.21037/tlcr-20-1134

\section{Clinical approach to oligometastatic and oligoprogressive NSCLC}

The definition of oligometastatic disease (OMD) in non-small cell lung cancer (NSCLC) is still rather heterogeneous (1): indeed, most of the studies limit such definition to the presence of no more than five metastatic sites, involving maximum two organs, while other involve patients with three or less metastatic sites. Nevertheless, it is important to notice that the majority of patients enrolled in dedicated clinical trials have a single metastasis $(2,3)$. From the analysis of a case-based survey involving 10 real-life patient cases discussed in a multidisciplinary context by a panel of NSCLC experts, a conservative OMD definition emerged, which include number of organs involved, mediastinal nodes status and prospects for radical treatment, as crucial components for daily practice decision-making (4). A consensus statement about the definition and staging of OMD NSCLC was then formulated by a pan-European multidisciplinary group: a maximum of five individual metastases (excluding the primary site), involving a maximum of three organs, was proposed; fluorodeoxyglucose F18-labeled positron emission tomography and computed tomography (FDG PET-CT) and brain assessment were recommended as mandatory, while biopsy and/or additional dedicated imaging were proposed in selected cases, usually after multidisciplinary team discussion (5). Recently, a consensus involving experts from the European Organisation for Research and the Treatment of Cancer (EORTC) and European Society for Radiotherapy and Oncology (ESTRO) has developed an oligometastatic disease classification system which should be applied to all cancer patients treated with local ablative therapy (LAT). Furthermore, the authors proposed a dynamic oligometastatic state model, underlying the dynamic transitions between oligorecurrent, oligoprogressive, and oligopersistent disease, depending on the response to local and systemic therapy (6).
The timing of metastases appearance seems to have a crucial impact in terms of prognosis. Patients affected by metachronous OMD without nodal involvement are considered at "low-risk" and experience a better overall survival (OS; 5-year OS 47.8\%), while synchronous metastases with one or more positive lymph nodes (N1/N2) define the patient at "high-risk" of developing further metastases with a worse prognosis (5-year OS $13.8 \%$ ), as defined by an individual patient data metanalysis (7). Nevertheless, both groups appear to benefit from local therapies.

Another subgroup of NSCLC that may have a prognostic benefit from an ablative treatment (usually surgery or radiotherapy) for one or more metastatic sites includes patients who develop disease progression in one or a limited number of metastatic sites, after achieving disease stability or objective response to systemic treatment. Such entity is widely recognized as oligoprogressive disease (OPD) and its biological behavior is likely to be related to tumor heterogeneity, which, under the selective pressure of the applied systemic treatment, promotes the development of one or more clones harboring intrinsic resistance mechanisms, and/or to the crosstalk between cancer cells and the surrounding tumor microenvironment (TME), whose behavior can also be modulated in a pro- or antitumor fashion by the ongoing treatment (8).

Although physicians should be aware that patients affected by OMD may benefit from a more aggressive approach (local therapies) than the "classical" stage IV NSCLC patients, at this time the only recognized tool to define oligometastatic disease is through imaging as a surrogate and still controversial biomarker for low metastatic potential. Initial studies aimed at assessing the potential role of ablative therapies on outcome of OMD NSCLC, have focused on patients with isolated brain or adrenal metastasis, showing better OS with local treatment (9). Most of these studies combined systemic treatment and local consolidative therapy, stereotactic body radiation therapy (SBRT) in particular, demonstrating a significant improvement in both 
progression free survival (PFS) and OS, as compared with systemic treatment/observation alone $(10,11)$. Local ablative treatment of residual or synchronous oligometastatic sites in patients affected by NSCLC harboring epidermal growth factor receptor (EGFR) mutations treated with tyrosine kinase inhibitors (TKIs) appears to be safe and feasible with promising survival outcome $(12,13)$. Similar encouraging results were observed in oncogene-addicted lung cancer experiencing OPD during TKI treatment $(14,15)$. Studies encompassing surgical resection of oligometastatic sites, which may give deeper insights into the biology and underlying mechanisms of OMD/OPD NSCLC, are currently ongoing.

Most of the available evidence exploring the potential role of TME interactions in NSCLC was obtained regardless of the specific pattern and timing of disease progression; indeed, to the best of our knowledge, this review represents the first attempt at systematically analyze available data about the role of NSCLC/TME interactions in the specific setting of OMD/OPD.

We present the following article in accordance with the Narrative Review reporting checklist (available at http:// dx.doi.org/10.21037/tlcr-20-1134).

\section{Materials and methods}

A comprehensive PubMed and ClinicalTrials.gov search was performed on July/August 2020, to identify the published and ongoing studies exploring the role of TME in oligometastatic/oligoprogressive NSCLC. The following keywords were used: tumor microenvironment, TME, NSCLC, oligoprogressive, oligometastatic, oncogene addicted, immunotherapy, LAT. In order to acquire a complete and in-depth perspective on this emerging topic, all original articles and reviews investigating the role of TME in (oligo)progression and (oligo)metastasis were considered. Abstracts not published in extenso, case reports and non-English full texts were excluded. All inclusion criteria were evaluated in title, abstract, and full text of original papers, by two independent reviewers.

\section{Role and composition of TME in NSCLC genesis and progression}

In recent years TME has emerged as a crucial determinant of cancer onset, biological behavior, and progression (16).

NSCLC microenvironment is characterized by: (I) rich angiogenesis, providing an efficient pro-tumoral oxygen supply, and (II) florid immune environment composed by cytokines and immune cells and usually related to the chronic exposure of lung tissue to inhalant toxic agents, mainly cigarette smoking (17). Immune response occurring in the respiratory tract includes prompt phagocytosis of inhalant pathogens and particles, recognized by alveolar macrophages, the most represented leukocytes in lower airways, through pattern recognition receptors (PRRs) $(18,19)$. In addition, epithelial cells of the respiratory tract contribute to immune response not only by exerting a mechanical function, but also modulating secondary cytokines and chemokines (IL-1 $\beta$, IL-6, -7, -8, TNF- $\alpha$ ), reactive oxygen species, adhesion molecules, and other lung-specific factors such as surfactant proteins and $\beta$-defensins, in response to specific cytokines, such as interferon- $\gamma($ INF- $\gamma$ ) and IL1- $\beta$ (20). Of note, lung tissue exposed to cigarette smoking is usually enriched in myeloidderived suppressor cells (MDSC)-like cells, lacking the immunosuppressive functions that they acquire after tumor development (21). Finally, CD8+ and CD4+ T cells (with a predominance of the T-helper 1 subtype expressing INF- $\gamma$ ) and dendritic cells, whose recruitment and maturation is stimulated by granulocyte-macrophage colony-stimulating factor (GM-CSF) produced by CD4+ T-cells, are also present in lung tissue $(22,23)$.

\section{TME in lung cancer progression and metastatization}

The role of TME in cancer progression is widely established. Metastatic spread represents a multi-step process, based on a series of intricate interactions between cancer cells and various TME components. The metastatic potential of tumor cells depends on both the chemotactic factors secreted by the target organ and the intrinsic predisposition of neoplastic cells to home, proliferate and survive in that specific site $(17,24)$.

The first step of the metastatic process consists in the growth of the primary mass, invading local tissues (Figure 1). Degradation of the basement membrane, due to the coordinated action of integrins, proteases and adhesion molecules (L1CAM, L1 cell adhesion molecule, and CRMP1, collapsin response mediator protein 1) represents a crucial event at this stage $(25,26)$. At the same time, cancer cells gain a migratory phenotype through shape modification (becoming more spindle-shaped), loss of polarity, and adhesion molecules' switch from E-cadherin to $\mathrm{N}$-cadherin and vimentin (27). This process is universally recognized as "epithelial-to-mesenchymal transition" 


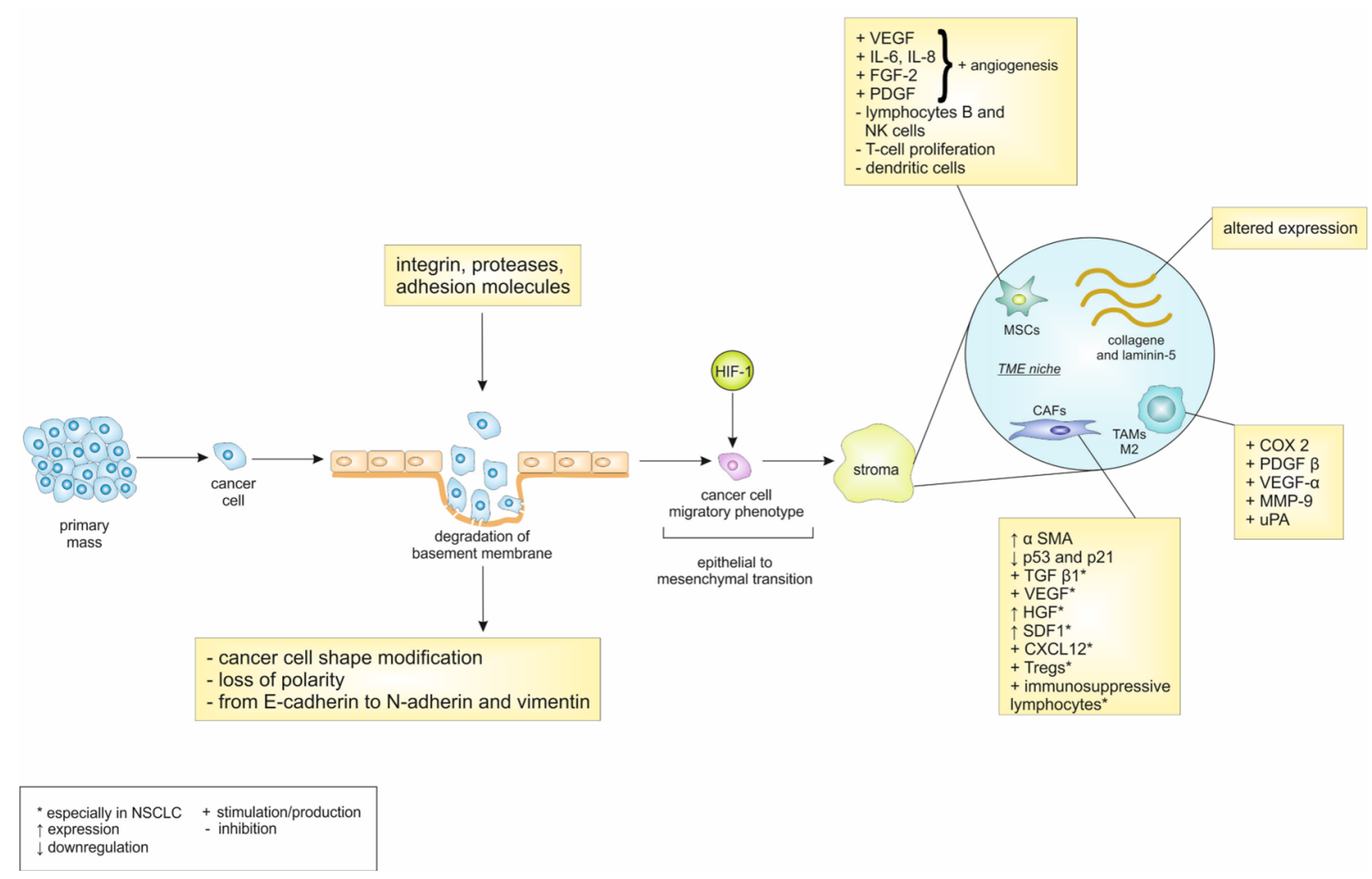

Figure 1 Local invasion of the stroma and composition of tumor microenvironment. HIF-1, hypoxia-inducible factor 1; TME, tumor microenvironment; MSCs, mesenchymal stem cells; VEGF, vascular endothelial growth factor; IL-6 and IL-8, interleukin-6 and interleukin-8; FGF-2, fibroblast growth factor; PDGF, platelet-derived growth factor; TAMs M2, tumor-associated macrophages M2; COX-2, cyclooxygenase-2; PDGF- $\beta$, platelet-derived growth factor beta; VEGF- $\alpha$, vascular endothelial growth factor alpha; MMP-9, matrix metalloproteinase-9; uPA, urokinase-type plasminogen activator; $\alpha$ SMA, alpha smooth muscle actin; TGF $\beta-1$, transforming growth factor beta-1; HGF, hepatocyte growth factors; SDF1, stromal cell-derived factor 1; CXCL12, C-X-C motif chemokine 12; Tregs, regulatory T cells.

(EMT) and is strongly induced, among other stimuli, by the hypoxic microenvironment created by the rapid growth of the primary tumor mass not balanced by efficient angiogenesis; hypoxia leads to the accumulation of hypoxiainducible factor 1 (HIF-1), which in turns promotes the transcription of EMT genes (28).

Once they have invaded the stroma, tumor cells interact with extracellular matrix (ECM) and stromal cells, thereby creating a microenvironment that is similar to the one of the wound healing $(17,29)$, with cancer associated fibroblasts (CAFs) as the predominant cell type able to promote tumor growth, modulate drug response and mediate protease-mediated ECM degradation (30). CAFs express high levels of alpha smooth muscle actin ( $\alpha \mathrm{SMA}$ ) and downregulate $\mathrm{p} 53$ and $\mathrm{p} 21$ (stress-response genes) $(31,32)$. CAFs and tumor cells produce transforming growth factor beta-1 (TGF- $\beta 1$ ), which has an ambivalent biological role: in early stages of tumor growth it exerts growth inhibitory functions, while in advanced stages it promotes tumor growth and progression $(33,34)$, through increased expression of anti-apoptotic factors (as Bcl-2) and modulation of the expression of adhesion molecules and integrins (35). In lung adenocarcinoma, CAFs have been confirmed to produce TGF- $\beta$, vascular endothelial growth factor (VEGF), and other pro-metastatic factors like hepatocyte growth factors (HGF) and stromal cell-derived factor 1 (SDF1), also known as C-X-C motif chemokine 12 (CXCL12) $(36,37)$, as well as to induce regulatory T cells (Tregs) and immunosuppressive lymphocytes (38).

Another cell population playing a crucial role in NSCLC invasion and metastasis is represented by the mesenchymal stem cells (MSCs) (39), which sustain an immunosuppressive status by reducing dendritic cells maturation (40), T-cell proliferation, B-lymphocytes 


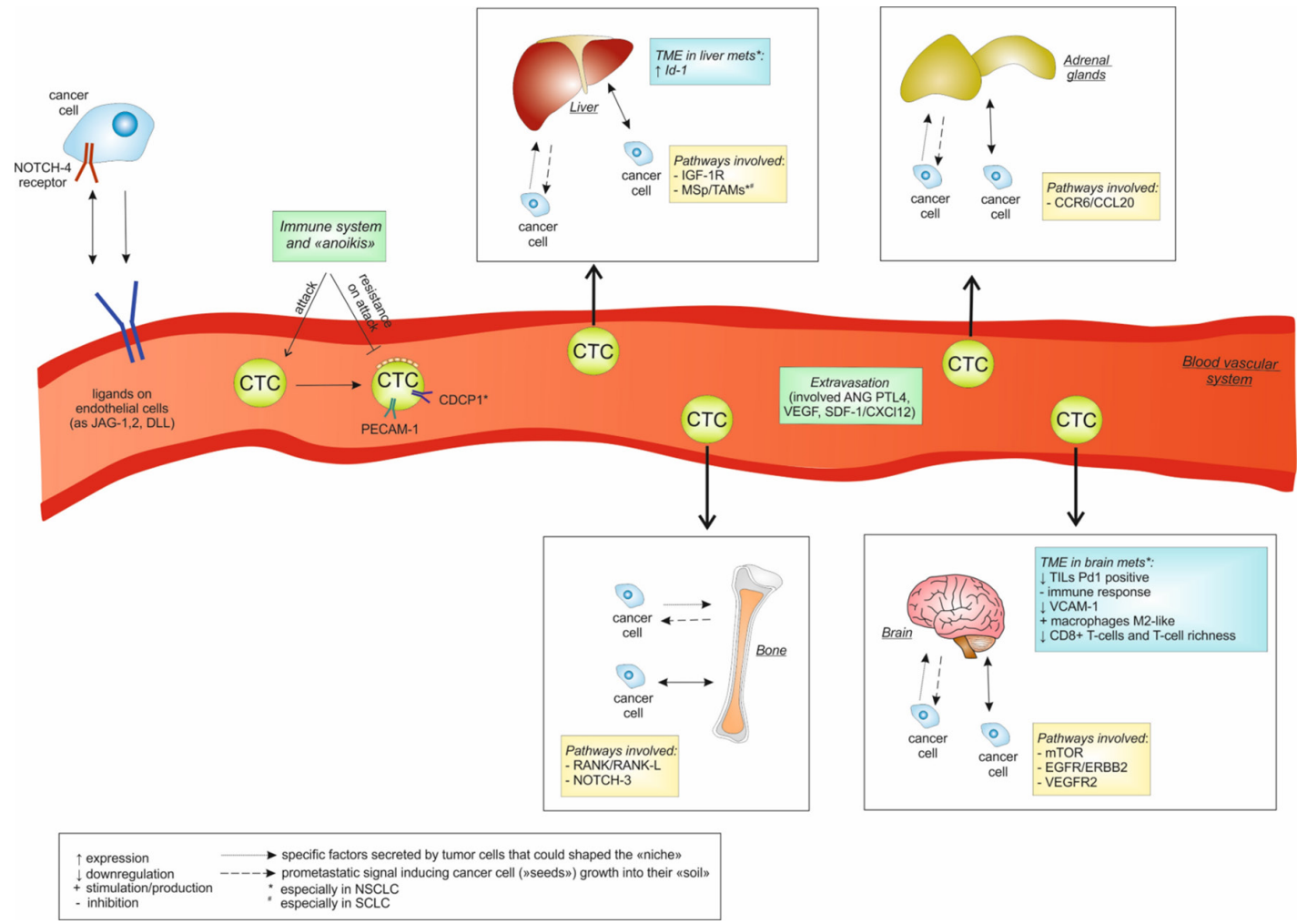

Figure 2 Intravasation/extravasation of cancer cells and "seed and soil" theory. JAG-1,2, Jagged ligands 1,2; DLL, delta-like ligand; CTC, circulating tumor cell; RANK/RANK-L, receptor activator of nuclear factor kappa-B/receptor activator of nuclear factor kappa-B ligand; PECAM-1, the platelet and endothelial cell adhesion molecule-1; CDCP1, CUB domain containing protein 1; TME, tumor microenvironment; mets, metastases; ANGPTL4, angiopoietin-like factor 4; VEGF, vascular endothelial growth factor; SDF1, stromal cell-derived factor 1; CXCL12, C-X-C motif chemokine 12; IGF-1R, insulin-like growth factor receptor 1; MSp/TAMS, macrophagestimulating protein/tumor-associated macrophages; mTOR, mammalian target of rapamycin; EGFR/ERBB2, epidermal growth factor receptor/receptor tyrosine-protein kinase erbB-2; VEGFR-2, vascular endothelial growth factor receptor 2; TILs PD-1 positive, PD-1positive tumor infiltrating lymphocytes; VCAM-1, vascular cell adhesion molecule-1; CCR6/CCL20, chemokines.

and NK cells activation (41). Moreover, MSCs stimulate angiogenesis, through the local production of factors such as platelet-derived growth factor (PDGF), fibroblast growth factor (FGF-2 and -6), IL-6, IL-8, VEGF, and angiopoietin-1 (17).

Finally, NSCLC stroma is particularly enriched in tumor-associated macrophages (TAMs), which can be divided into two main subtypes, M1 and M2, characterized by anti- and pro-tumorigenic properties, respectively. NSCLC cells recruit TAMs by secreting IL-17 and promote a prostaglandin E2 (PGE2)-mediated M2 switch (42). Protumorigenic M2 TAMs enhance tumor invasiveness by secreting cyclooxygenase-2 (COX2), PDGF-b, VEGF-a, HGF, cathepsin k, matrix metalloproteinase-9 (MMP-9) and urokinase-type plasminogen activator (uPA). The interaction between MMP-9 and uPA has a specific role in NSCLC invasion (43).

With regard to the interaction with ECM proteins, the expression of collagens and laminine-5 is altered in NSCLC, with the latter correlating with an upregulation in EGFR-AKT signaling (17). Lysyl-oxidase (LOX) is upregulated in response to hypoxia, enhancing tumor invasiveness by modulating focal adhesion kinase (FAK) signaling and creating cross-links among collagen fibers (44). Moreover, tumor cells directly modulate ECM structure and function, by increasing the production of specific MMPs (1, 2 and 9), whose polymorphisms have been associated with NSCLC risk and survival, suggesting the existence of a complex and bidirectional interaction between cancer cells and ECM $(45,46)$.

The second step of the metastatic process consists in the 
"intravasation" of cancer cells into the blood and lymphatic vascular systems to reach distant target organs (Figure 2). The specific mechanisms involved at this stage are not yet completely clarified, but two models have been proposed: a passive dissemination or an active mechanism following a cytokine gradient (17). Among the ligand-receptor pairs potentially involved, there are SDF1-CXCL12 and CXCR4CXCR7. The binding of CXCL12 to CXCR7, instead of CXCR4, reduces the expression of MMP12 and increases VEGF $\alpha$, stimulating the angiogenic pathway $(47,48)$. Furthermore, NSCLC cells express NOTCH1-4 receptors, which binding to their ligands JAG-1 and -2, DLL (deltalike) $-1,-3$ and -4 on stromal endothelial cells can induce intravasation and trans-endothelial migration (49).

Once passed through the blood vessel walls, circulating tumor cells (CTCs) are exposed to multiple threats, including immune recognition and immune-mediated elimination. To avoid immune attack, After entering the bloodstream CTCs are rapidly covered by an activated platelets layer (50), which hides antigen presenting domains with L- and P-selectins and tissue factor (51,52). Another mechanism involved in CTCs removal is a phenomenon called "anoikis", an apoptotic response to the loss of interaction between ECM and integrins (53). Metastatic NSCLC express markers of anoikis-resistance, such as the platelet and endothelial cell adhesion molecule-1 (PECAM-1) and CUB domain containing protein 1 (CDCP1) (54). Of note, metastatic cancer cells exist in the bloodstream also as disseminated tumor cells (DTC) and circulating tumor microemboli (CTM), whose biological significance and function are not yet well characterized (17).

Once at their destination, CTCs need to extravasate through the well-developed and almost impermeable host vessel walls (17). Cancer cells extravasation is an active process, comparable to the diapedesis of leucocytes, and involves several players, such as angiopoietin-like factor 4 (ANGPTL4) (55), VEGF and SDF-1/CXCL12 (56), as well as adhesion molecules (57). The destination of CTCs is not only influenced by "mechanical" factors, such as the direction of the blood flow: validated models propose that the microenvironment of target tissues produce prometastatic signals inducing cancer cells (the "seeds") to survive and growth into their new "soil" (58). One underlying mechanism assumes the existence of a premetastatic niche, produced in response to specific factors primarily secreted by the tumor (59). Such niche has a fibrotic nature and contains a high number of activated fibroblasts and VEGFR-positive immature myeloid cells, able to create and maintain a suitable environment for adhesion and invasion. Recent evidence suggest that such "niche" could be shaped by both the primary tumor and MSCs through the induction of an immunosuppressive microenvironment, favored, for example, by the expression of programmed death ligand-1 (PD-L1), which binds to PD-1 on macrophages, $\mathrm{T}$ and $\mathrm{B}$ cells thus inhibiting their activation, and the recruitment of suppressive cells as TAMs, tumor associated neutrophils (TANs) and Tregs (60).

In terms of target organs, it is likely that metastatic cells show a selective tropism, dependent on the activation of specific pathways and the expression of adhesion molecules. In NSCLC, the most frequent sites of metastatization are represented by liver (33-40\%), brain (15-43\%), bone (19$33 \%)$ and adrenal glands (18-38\%) (61).

Brain metastases seem to follow a CXCL12 gradient and show the activation of CXCR4/CXCL12-mediated antiapoptotic pathways $(62,63)$. Signaling pathways strongly involved in brain metastases include mTOR, EGFR/ERBB2, and VEGFR2 (64). Moreover, available studies support the existence of a more immunosuppressive microenvironment in brain metastases, as compared to the primary tumor, potentially related to several factors: (I) limited quantity of PD-1-positive tumor infiltrating lymphocytes (TILs) (65); (II) inhibition of pathways involved in leukocyte extravasation, dendritic cell maturation, and Th1 immune responses; (III) reduced expression of adhesion molecules, such as vascular cell adhesion molecule-1 (VCAM-1); (IV) presence of pro-tumorigenic M2-like macrophages; (V) reduced CD8+ T cell and TILs infiltration and (VI) limited T-cell richness and density (66).

Bone metastases from NSCLC are typically osteolytic, induced by the interaction between osteoblasts and osteoclasts with the crucial mediation of receptor activator of nuclear factor kappa-B ligand (RANKL), which promotes bone degradation and thus the release of ECM factors, further attracting metastatic cells. A preclinical study suggested that increased expression of RANK, RANKL and osteoprotegerin (OPG) is correlated with a higher metastatic potential to the bone; these proteins are frequently detected in bone metastases from NSCLC. In this light, a direct production of RANKL from metastatic sites may further facilitate the migration of RANK-positive cancer cells (67). NF- $\mathrm{kb}$, a crucial mediator of the RANKL pathway, can also be activated by EGFR and KRAS signaling, frequently boosted in NSCLC, through the phosphorylation of I $\kappa \mathrm{B}$, an inhibitory protein that segregates NF-kb into the cytosol. Therefore, NF-kb can dimerize, translocate into the nucleus and act as a transcription factor for several genes involved 
in the inflammation process, innate and adaptive immune response, and carcinogenesis (68). In addition, an increased expression of Notch-3 contributes to TGF- $\beta$-driven EMT and to development of bone metastasis in NSCLC (69).

Increased expression of insulin-like growth factor receptor 1 (IGF-1R) represents one of the main potential "triggers" of metastatic spread to the liver. IGF-1R signaling is involved in DNA synthesis, anchorage-independent cell growth, apoptosis, cell migration and tissue invasion (70), as well as in the production of altered collagen IV, III and XVIII (71). Integrin alpha2 binding to collagen IV has been associated to anoikis resistance and hepatic metastatization (72). The chemotactic factor macrophage-stimulating protein (MSP) promotes liver metastases when overexpressed in small-cell lung cancer (SCLC) and induces liver recruitment of TAMs producing pro-angiogenic growth factors (73). Finally, NSCLC-related liver metastases usually express high levels of inhibitor of differentiation (Id-1), whose suppression inhibits TGF $\beta$ expression and EMT (74).

Biological mechanisms underlying adrenal gland metastatization are still unclear. Ligand-receptor complex CCR6/CCL20 is likely to be involved, as CCR6 has been found overexpressed in adrenal metastases from lung cancer (75).

\section{Role of TME at progression during TKIs or immunotherapy}

\section{TME and progression to anti-EGFR TKIs}

Given the clinical implications of progressive disease in EGFR-mutant NSCLC, TME status at the time of progression to anti-EGFR TKIs has been investigated. Tumor cells produce molecules able to promote cell growth regardless of EGFR activation, such as IL-6, growth-arrest specific protein-6 (GAS6), HGF and EGF, in an autocrine manner (76). Alterations in cell-to-cell adhesion are also involved, with increased levels of $\mathrm{N}$-cadherin and integrin $\beta 1$ and suppression of Serpin B2, thus resulting in a more invasive behavior due to ECM degradation $(77,78)$. CAFs and MSCs express several molecules (as CXCR4, IL6-R, HGF, AXL, TGF $\beta-R$ ) involved in TKIs resistance through EMT promotion, enhanced cell survival by MAPK and JAK-STAT signaling, and apoptosis inhibition through increased Bcl-2 activity (76). The interaction between HGF and mesenchymal-epithelial transition factor (MET) plays a crucial role in mediating EGFR-TKIs resistance. Available data reported that the CAFs of TKI-resistant tumors express higher levels of HGF and that the addition of an anti-HGF antibody may restore response to TKI in vitro (79). In clinical practice, MET targeting represents a crucial strategy in trying to overcome MET-driven resistance to EGFR-TKIs (80).

EMT is another crucial mechanism of resistance, mediated by the autocrine and paracrine action of TGF- $\beta$ (81), leading cancer cells to progressively lose the epithelial markers, while increasing expression of the mesenchymal ones (as vimentin) (82). As discussed above, a hypoxic environment promotes HIF- $1 \alpha$ expression by cancer cells, inducing TKI resistance through activation of the TGF $\alpha-I G F R 1$ pathway and enhanced angiogenesis mediated by VEGF secretion $(83,84)$.

Immune cells involved in TKIs resistance are mainly TAMs, producing VEGF and promoting activation of MAPK, NF- $\mathrm{B}$, TGF- $\beta$ and RAS pathways (85). A retrospective study including 70 patients treated with antiEGFR TKIs reported relevant prognostic differences according to TME composition with a worse outcome for the combined presence of high PD-L1 tumor expression and high levels of CD8+ TILs [median PFS 2.4 months, response rate (RR) $14.3 \%$ ] (86).

\section{TME and progression to anti-ALK TKIs}

Regarding resistance to anaplastic lymphoma kinase (ALK) inhibitors, available data suggest that the activation of EGFR and MET pathways can mediate cell growth, bypassing the oncogenic ALK signaling (87). Hypoxia as well may induce EMT and resistance to ALK inhibitors in ALK-rearranged NSCLC (88).

\section{TME and progression to immunotherapy}

Immune checkpoint inhibitors (ICIs) targeting PD-1/ PD-L1, both in monotherapy and in combination with chemotherapy, have demonstrated to remarkably improve overall survival in locally advanced and metastatic lung cancer (89). Nevertheless, response to ICIs is hardly predictable, with PD-L1 expression being the only validated predictive biomarker so far. In order to clarify the individual response to ICIs, tumors have been stratified according to the presence of immune cells in their TME. This has led to a first splitting into "hot" and "cold" tumors. "Hot" or inflamed tumors are characterized by strong T-cell infiltration (particularly composed of CD8+ lymphocytes), high genomic instability (resulting in a high amount of neoantigens), and a pre-existing immune response with high levels of chemokines (such as CCL5, CXCL9, CXCL10) 
recruiting T-cells and a strong expression of IFN- $\gamma$ to support their anti-tumor function. On the other hand, "cold" tumors show poor T-CD8+ infiltration, with high amounts of macrophages and fibroblasts and a low tumor mutational burden $(90,91)$. Moreover, TGF- $\beta$ prevents response to anti PD-L1 and leukemia inhibitory factor (LIF) cytokine inhibits the production of T-cell attracting chemokines (90). A third phenotype has been proposed, defined as "altered", further divided into "excluded" tumors, where immune cells are confined at the edge of the tumor with no infiltration signs, and "immune-suppressed" tumors, with a low degree of immune cells reflecting an immunosuppressive microenvironment (91). The overexpression of tryptophan metabolites, particularly kynurenine, produced by the indoleamine 2,3-dioxygenases (IDO-1), has been associated with an immune-suppressive TME (92). A preliminary study conducted on 26 patients treated with ICIs reported an enhanced activity of this enzyme in the subgroup experiencing early disease progression, while a lower kynurenine/tryptophan ratio correlated with better outcome $(92,93)$.

A rich TME infiltration by TILs (CD8+ and CD4+) has been associated with a favorable prognosis in different tumors, including NSCLC (94). Currently, a growing body of data suggests that specific phenotypes of immune cells and the diversity of immune repertoire might correlate with ICIs efficacy (95). In detail, the TCF7+/TCF7- CD8+ ratio is predictive of response and survival in melanoma patients treated with ICIs (96). In another study, differential expression of CD39 between two populations of tumorinfiltrating CD8+ was associated to better recognition of tumor-associated antigens (97). The emerging role of TILs in lung cancer microenvironment, alongside with a high clonal mutation burden due to carcinogen exposure, are leading to the development of adoptive T-cell therapy approaches, using autologous TILs enriched with T cells specifically recognizing clonal neoantigenic epitopes by selective expansion; such approach may offer a further chance to patients who become resistant to anti PD1/PDL1 therapy (98).

Peripheral blood immune cell populations, reflecting tumor immune microenvironment, are currently under investigation as putative predictive and prognostic factors, potentially suitable for non-invasive immune monitoring. MDSCs are an immature population of myeloid cells with a strong immunosuppressive activity, inhibiting in particular T- and NK-cell functions (99). In this light, high numbers of circulating or infiltrating MDSCs may portend worse prognosis and reduced response to ICIs in NSCLC and other cancer types. The interaction between TME and ICIs is reciprocal, however, as MDSCs levels and function are, in turn, influenced by ICIs. Bertelli et al. reported a case of strong reduction in MDSC1 (a CD14+ and CD124+ subset of MDSC) number and suppressive activity in a NSCLC patient treated with durvalumab after chemo-radiotherapy, clinically and radiologically corresponding to longlasting disease remission (100). Probably the most deeply investigated blood biomarker is the neutrophil/lymphocyte ratio (NLR), or the dNLR [neutrophils-(leucocytesneutrophils)], whose value at baseline appears to correlate with response to ICIs. A recent metanalysis has confirmed that NSCLC patients treated with ICIs have poorer OS and PFS when their baseline NLR or dNLR is high (101). Together with baseline LDH, dNLR constitutes the Lung Immune Prognostic Index (LIPI): a poor LIPI score ( $d N L R \geq 3$ and LDH $>$ ULN) is associated with poor PFS, OS and disease control rate, as compared to an intermediate $(\mathrm{dNLR}>3$ OR LDH $>$ ULN) or good (dNLR $<3$ and LDH $<\mathrm{ULN}$ ) score, in a retrospective analysis of 466 patients treated with ICIs; conversely, LIPI stratification was not predictive of response to chemotherapy (102).

The role of gut microbioma, recently defined as a key component of TME (103), in ICIs response is yet to be clarified. The presence and health of gut microbioma are of primary importance in the development of both a local (gut lymphoid tissue, lamina propria CD4+ cells, IgA production) and systemic immunity, as many bacterial-derived molecules [as lipopolysaccharide (LPS), peptidoglycan, flagellin] enhance the activity and correct maturation of innate immune cells such as dendritic cells, macrophage and neutrophils. In mice, adaptive immunity has been demonstrated to depend on gut bacteria as well, as germ-free mice poorly developed B follicles and T-cell zones within spleen and lymph nodes (104). An analysis performed on 89 melanoma patients treated with anti-PD1 showed a more efficient antigen presentation and effector $\mathrm{T}$ function in the subgroup with a higher heterogeneity in gut microbioma composition, while patients with a lower diversity had limited intratumoral lymphoid and myeloid infiltration and weakened antigen presentation capacity, conditioning a worse response to ICIs (95). Microbioma heterogeneity seems to be affected by antibiotic therapy. Derosa et al. retrospectively reported a significant decrease in PFS and OS in NSCLC patients who received antibiotics, particularly within the first 30 days from ICIs start (105). Microbioma composition was evaluated in a study involving NSCLC and renal cell cancer receiving 
anti-PD-1 treatment. A prevalence of Akkermansia Muciniphila was associated with a better clinical outcome, as it was detectable in $69 \%$ of patients who responded to ICIs, along with a well-represented number of commensals such as Ruminococcus spp., Alistipes spp., and Eubacterium spp.; conversely, A. Muciniphila could only be detected in $34 \%$ of patients who progressed or died. When mice with antibiotic gut sterilization received fecal microbiota transplant with stool of responder patients or an oral gavage with $A$. Muciniphila, response to anti-PD1 was restored, demonstrating a crucial role of gut microbioma composition on the outcome to anti-PD1 therapy (106).

\section{TME in oligometastatic and oligoprogressive cancers, including NSCLC}

Despite the fact that local microenvironmental interactions are arguably crucial in determining metastatic development and therapy resistance in specific locations, limited data are available about the role of TME in oligometastatic and oligoprogressive solid tumours. The first attempt at characterizing factors potentially able to influence the rate of success or failure of oligo- and widespread metastases was reported by Reyes and Pienta $(24,107)$ : the "quality" of the primary microenvironment is dependent from multiple factors, including $\mathrm{pH}$, oxygenation, amount of nutrients, interaction with supporting host cells, and quality/quantity of immune response. Epithelial cancer cells growing into a highly vascularized environment, enriched in nutrients, are less likely to evolve towards a more aggressive behaviour through EMT, becoming more motile and leaving a relatively hostile original site; as the quality of the primary TME decreases, the generation of lethal, mesenchymal-like clones increases $(24,107)$.

Few studies explored the biology of oligometastases and oligoprogression in oncogene-addicted NSCLC, particularly in NSCLC cases harboring EGFR mutations (108). Metastatization patterns in EGFR-mutant NSCLC are often characterized by progression in a limited number of anatomic sites, such as the brain. These patterns of progression/metastatization are likely to represent a manifestation of intratumor and intertumor genomic heterogeneity (109-112). In 2008, Taniguchi et al. described the intratumor heterogeneity of EGFR mutations, reporting the presence of both EGFR-mutant and EGFR-wild type clones in 6 out of 21 patients treated with gefitinib (109). Moreover, discordant $E G F R / B R A F / K R A S$ mutational patterns were detected between primary tumors and lymph node metastases $(110,111)$. Chen et al. described, in paired samples of primary lung adenocarcinoma and regional/distant metastases, an overall discordance rate in EGFR mutation status of $13.9 \%$. Furthermore, the authors showed that a significant proportion of patients $(29 \%)$ who developed resistance to EGFR TKIs, had a significant heterogeneity between the primary tumor and metastatic sites or between individual metastatic sites (112). Whether and which local microenvironmental interactions may influence metastatic fitness and/or therapy resistance of specific tumour clones (e.g., EGFR-mutant versus $E G F R$ wild type) in different metastatic locations, however, remains to be established.

Preliminary data trying to unravel the biological bases of OMD/OPD in lung cancer are enriched by pivotal studies available in other solid tumors. An analysis performed on 335 matched metastases from 575 renal cell carcinoma samples suggested rapid progression and reduced OS in metastatic specimens harboring $9 p$ loss, low intratumor heterogeneity, and a higher proportion of genomic somatic copy-number alterations. In this study, the few patients experiencing "attenuated progression" (defined as the development of a single metastatic site within 6 months from surgery or multisite progression more than 6 months after surgery) were affected by tumors showing high intratumoral heterogeneity, low rate of somatic copy number alterations, PBRM1 and SETD2 driver mutations (113). Another study performed in patients undergoing partial hepatectomy for colorectal cancer metastases identified three main molecular subtypes ("classic", "immune" and "stromal") according to their peculiar molecular profile. Among them, the "stromal" cohort, associated with prevalent KRAS, EMT, and angiogenesis signaling pathways, was associated with a higher metastatic potential with an increased risk of developing extrahepatic metastatic disease. Interestingly, the "immune" molecular subtype, mainly characterized by high immune infiltration, interferon alpha and gamma signatures, activation of p53 pathways, overexpression of innate and adaptive immune genes, identified a relatively indolent subgroup which developed clinically evident metastases limited in number (oligometastatic state) (114).

An interesting finding is that microRNAs may have a role in mediating the metastatic potential. Khodarev et al. profiled microRNAs derived from cancer patients with oligo- or poly- lung metastases from different primary sites treated with SBRT or surgery, in order to examine their potential role in driving the oligometastatic phenotype. These microRNAs were defined as "oligomiRs". Among 
them, one common locus at $14 \mathrm{q} 32$ associated with carcinogenesis and stem cell functioning, encoded for a significant number of oligomiRs (115).

Notwithstanding the limited amount of available data about TME in oligometastatic disease, borrowing the available evidence from the metastatic setting may provide additional insights for clinical/translational studies in this setting. In particular, in the context of clinical trials, when a bioptic procedure is feasible, comparing the study of TME (i.e., exploring the composition in terms of immune cells such as fibroblasts, TAMs, immature myeloid cells and Tregs, and the expression of VEGF, PD-L1 and adhesion molecules) between primary tumor and oligometastatic site and between different oligometastatic sites may offer a chance to further deepen the knowledge about the mechanisms supporting the OMD process.

\section{Ongoing studies including LAT for oligometastatic and oligoprogressive NSCLC}

The real impact of TME in oligometastatic and oligoprogressive lung cancer still represents an untapped field, presumably because patients with only one or a few metastatic sites (at diagnosis or at progression) are often not considered candidate to surgery and routine re-biopsy does not represent the standard of care. As highlighted above, an increasing amount of data supports a potential positive impact of LAT, radiotherapy or surgery, in addition to systemic therapy, for OMD or OPD lung cancer patients. In the majority of ongoing trials, the collection of tissue/ cytological samples at baseline is mandatory, but only a few studies also collect surgical specimens deriving from the removal of OMD/OPD sites: in particular, only one study (NCT03827577) requires the surgical removal of both the primary tumor and/or all oligometastases. Studies encompassing the collection of tumor (and/or blood) material offer a concrete chance to deepen the current knowledge about TME in OMD/OPD lung cancer, possibly revealing a dynamic landscape of cell infiltration and genetic alterations able to drive the oligometastatic/ oligoprogressive process (116) (Table 1).

\section{Conclusions}

To date, the real impact and biological meaning of TME in OMD and OPD lung cancer is still far to be clarified.

Table 1 Ongoing clinical trials including TME evaluation in OMD/OPD lung cancer

\begin{tabular}{|c|c|c|c|c|c|c|c|c|}
\hline Phase & $\begin{array}{l}\text { ClinicalTrials. } \\
\text { gov identifier }\end{array}$ & Setting & $\mathrm{N}$ & Treatment & $\begin{array}{l}\text { Type of tumor } \\
\text { sample }\end{array}$ & $\begin{array}{l}\text { Primary } \\
\text { endpoints }\end{array}$ & $\begin{array}{l}\text { Secondary } \\
\text { endpoints }\end{array}$ & Status \\
\hline $\mathrm{Ilb}$ & NCT04216121 & $\begin{array}{l}\text { OPD EGFR-mu- } \\
\text { tant NSCLC }\end{array}$ & 39 & $\begin{array}{l}\text { LAT (SBRT or surgery) + first line } \\
\text { osimertinib }\end{array}$ & $\begin{array}{l}\text { Surgical } \\
\text { specimen }^{1}\end{array}$ & PFS2 & $\begin{array}{l}\text { Time to next line } \\
\text { systemic therapy; } \\
\text { patterns of } \\
\text { progression to } \\
\text { LAT; QoL }\end{array}$ & $\begin{array}{l}\text { Active, } \\
\text { recruiting }\end{array}$ \\
\hline II & NCT03808662 & $\begin{array}{l}\text { OPD triple nega- } \\
\text { tive breast cancer } \\
\text { or NSCLC }\end{array}$ & 160 & $\begin{array}{l}\text { SBRT to all the OPD sites vs. } \\
\text { SOC }\end{array}$ & Tissue biopsy² & PFS & os & $\begin{array}{l}\text { Active, } \\
\text { recruiting }\end{array}$ \\
\hline$\| / / I I I$ & NCT02759783 & $\begin{array}{l}\text { Extracranial OMD } \\
\text { prostate cancer, } \\
\text { breast cancer or } \\
\text { NSCLC }\end{array}$ & 245 & $\mathrm{SOC}+/-\mathrm{SBRT}$ & Tissue biopsy² & PFS & $\begin{array}{l}\text { OS, local lesion } \\
\text { control, } \\
\text { QoL, FFWMD }\end{array}$ & $\begin{array}{l}\text { Active, not } \\
\text { recruiting }\end{array}$ \\
\hline
\end{tabular}

Table 1 (continued) 
Table 1 (continued)

\begin{tabular}{|c|c|c|c|c|c|c|c|c|}
\hline Phase & $\begin{array}{l}\text { ClinicalTrials. } \\
\text { gov identifier }\end{array}$ & Setting & $\mathrm{N}$ & Treatment & $\begin{array}{l}\text { Type of tumor } \\
\text { sample }\end{array}$ & $\begin{array}{l}\text { Primary } \\
\text { endpoints }\end{array}$ & $\begin{array}{l}\text { Secondary } \\
\text { endpoints }\end{array}$ & Status \\
\hline II & NCT02316002 & OMD NSCLC & 51 & $\begin{array}{l}\text { Pembrolizumab after definitive } \\
\text { therapy }\end{array}$ & $\begin{array}{l}\text { Tissue biopsy } \\
\text { or surgical } \\
\text { specimen }^{2}\end{array}$ & PFS & & $\begin{array}{l}\text { Active, not } \\
\text { recruiting }\end{array}$ \\
\hline III & NCT03391869 & Stage IV NSCLC & 270 & Nivolumab + ipilimumab +/- LCT & $\begin{array}{l}\text { Tissue biopsy } \\
\text { or cytological } \\
\text { sample }^{2}\end{array}$ & $\begin{array}{l}\text { OS; OS in } \\
\text { OMD } \\
\text { subgroup }\end{array}$ & PFS, TANM & $\begin{array}{l}\text { Active, } \\
\text { recruiting }\end{array}$ \\
\hline III & NCT02076477 & OMD NSCLC & 420 & $\begin{array}{l}\mathrm{CT}(\text { cisplatin + docetaxel or } \\
\text { pemetrexed })+\mathrm{RT}\end{array}$ & $\begin{array}{l}\text { Tissue biopsy } \\
\text { or cytological } \\
\text { sample }^{2}\end{array}$ & $\mathrm{RR}$ & PFS & $\begin{array}{l}\text { Active, } \\
\text { recruiting }\end{array}$ \\
\hline II & NCT03965468 & OMD NSCLC & 47 & $\begin{array}{l}\text { Durvalumab + } \\
\text { carboplatin-paclitaxel + SBRT on } \\
\text { all the metastatic sites + surgery } \\
\text { or radical RT on the primary }\end{array}$ & $\begin{array}{l}\text { Surgical } \\
\text { specimen }^{1}\end{array}$ & PFS & $\begin{array}{l}\text { OS, OR, DoR, } \\
\text { distant PFS }\end{array}$ & $\begin{array}{l}\text { Active, } \\
\text { recruiting }\end{array}$ \\
\hline III & NCT03827577 & OMD NSCLC & 195 & $\begin{array}{l}\text { SOC +/- LAT of all metastatic } \\
\text { sites and resection of the } \\
\text { primary }\end{array}$ & $\begin{array}{l}\text { Surgical } \\
\text { specimen }^{3}\end{array}$ & Os & & $\begin{array}{l}\text { Active, } \\
\text { recruiting }\end{array}$ \\
\hline
\end{tabular}

1, surgical specimen available if LAT is performed with surgery of the oligometastatic sites; ${ }^{2}$, baseline tissue biopsy, cytological sample or surgical specimen (either on primary tumor or on metastasis); ${ }^{3}$, surgical specimen of primary and/or of all oligometastases. N, number; OPD, oligoprogressive disease; NSCLC, non-small cell lung cancer; LAT, local ablative therapy; SBRT, stereotactic body radiotherapy; PFS, progression free survival; PFS2, progression free survival (at second progression); QoL, quality of life; RT, radiotherapy; RR, response rate; OS, overall survival; SOC, standard of care; OMD, oligometastatic disease; ORR, overall response rate; FFWMD, freedom from widespread metastatic disease; LCT, Local Consolidation Therapy; TANM, Time to Appearance of New Metastases; CT, chemotherapy; OR, overall response; DoR, duration of response.

Ongoing trials exploring the efficacy of LAT in this setting, in particular those involving surgery as the main therapeutic approach to treat oligometastases, may help to provide crucial information. Deepening the knowledge on TME composition and function in OMD/OPD may provide innovative implications in terms of both prognosis and prediction of outcome from local treatments. Moreover, new candidate player(s) able to drive and/or support oligometastatization or oligo progression may emerge, paving the way for future investigations of targeted therapeutic approaches in both advanced and early disease settings.

\section{Acknowledgments}

Funding: EB is supported by Institutional funds of
Università Cattolica del Sacro Cuore (UCSC-project D12019-2020). EB is currently supported by the Associazione Italiana per la Ricerca sul Cancro (AIRC) under Investigator Grant (IG) No. IG20583.

\section{Footnote}

Provenance and Peer Review: This article was commissioned by the Guest Editors (Maurizio Infante \& Thierry Berghmans) for the series "Oligometastatic NSCLC: definition and treatment opportunities" published in Translational Lung Cancer Research. The article has undergone external peer review.

Reporting Checklist: The authors have completed the Narrative Review reporting checklist. Available at http:// 
dx.doi.org/10.21037/tlcr-20-1134

Conflicts of Interest: All authors have completed the ICMJE uniform disclosure form (available at http://dx.doi. org/10.21037/tlcr-20-1134). The series "Oligometastatic NSCLC: definition and treatment opportunities" was commissioned by the editorial office without any funding or sponsorship. EB serves as an unpaid editorial board member of Translational Lung Cancer Research from September 2019 to September 2021. MM reports honoraria or speakers' fee from Pfizer, EUSA Pharma and Astra Zeneca, outside the submitted manuscript. SP reports honoraria or speakers' fee from Astra-Zeneca, Eli-Lilly, BMS, Boehringer Ingelheim, MSD and Roche, outside the submitted manuscript. The authors have no other conflicts of interest to declare.

Ethical Statement: The authors are accountable for all aspects of the work in ensuring that questions related to the accuracy or integrity of any part of the work are appropriately investigated and resolved.

Open Access Statement: This is an Open Access article distributed in accordance with the Creative Commons Attribution-NonCommercial-NoDerivs 4.0 International License (CC BY-NC-ND 4.0), which permits the noncommercial replication and distribution of the article with the strict proviso that no changes or edits are made and the original work is properly cited (including links to both the formal publication through the relevant DOI and the license). See: https://creativecommons.org/licenses/by-nc-nd/4.0/.

\section{References}

1. Giaj-Levra N, Giaj-Levra M, Durieux V, et al. Defining Synchronous Oligometastatic Non-Small Cell Lung Cancer: A Systematic Review. J Thorac Oncol 2019;14:2053-61.

2. Laurie SA, Banerji S, Blais N, et al. Canadian consensus: oligoprogressive, pseudoprogressive, and oligometastatic non-small-cell lung cancer. Curr Oncol 2019;26:e81-e93.

3. Planchard D, Popat S, Kerr K, et al. Correction to: "Metastatic non-small cell lung cancer: ESMO Clinical Practice Guidelines for diagnosis, treatment and followup". Ann Oncol 2019;30:863-70.

4. Hendriks LEL, Dooms C, Berghmans T, et al. Defining oligometastatic non-small cell lung cancer: A simulated multidisciplinary expert opinion. Eur J Cancer
2019;123:28-35.

5. Dingemans AC, Hendriks LEL, Berghmans T, et al. Definition of Synchronous Oligometastatic Non-Small Cell Lung Cancer-A Consensus Report. J Thorac Oncol 2019;14:2109-19.

6. Guckenberger M, Lievens Y, Bouma AB, et al. Characterisation and classification of oligometastatic disease: a European Society for Radiotherapy and Oncology and European Organisation for Research and Treatment of Cancer consensus recommendation. Lancet Oncol 2020;21:e18-e28.

7. Ashworth AB, Senan S, Palma DA, et al. An individual patient data metaanalysis of outcomes and prognostic factors after treatment of oligometastatic non-small-cell lung cancer. Clin Lung Cancer 2014;15:346-55.

8. Tumati V, Iyengar P. The current state of oligometastatic and oligoprogressive non-small cell lung cancer. J Thorac Dis 2018; 10:S2537-44.

9. Counago F, Luna J, Guerrero LL, et al. Management of oligometastatic non-small cell lung cancer patients: Current controversies and future directions. World J Clin Oncol 2019;10:318-39.

10. Gomez DR, Tang C, Zhang J, et al. Local Consolidative Therapy Vs. Maintenance Therapy or Observation for Patients With Oligometastatic Non-Small-Cell Lung Cancer: Long-Term Results of a Multi-Institutional, Phase II, Randomized Study. J Clin Oncol 2019;37:1558-65.

11. Palma DA, Olson R, Harrow S, et al. Stereotactic ablative radiotherapy versus standard of care palliative treatment in patients with oligometastatic cancers (SABRCOMET): a randomised, phase 2, open-label trial. Lancet 2019;393:2051-8.

12. Chan OSH, Lam KC, Li JYC, et al. ATOM: A phase II study to assess efficacy of preemptive local ablative therapy to residual oligometastases of NSCLC after EGFR TKI. Lung Cancer 2020;142:41-6.

13. Xu Q, Zhou F, Liu H, et al. Consolidative Local Ablative Therapy Improves the Survival of Patients With Synchronous Oligometastatic NSCLC Harboring EGFR Activating Mutation Treated With First-Line EGFRTKIs. J Thorac Oncol 2018;13:1383-92.

14. Weickhardt AJ, Scheier B, Burke JM, et al. Local ablative therapy of oligoprogressive disease prolongs disease control by tyrosine kinase inhibitors in oncogene-addicted nonsmall-cell lung cancer. J Thorac Oncol 2012;7:1807-14.

15. Yu HA, Sima CS, Huang J, et al. Local therapy with continued EGFR tyrosine kinase inhibitor therapy as a treatment strategy in EGFR-mutant advanced lung cancers 
that have developed acquired resistance to EGFR tyrosine kinase inhibitors. J Thorac Oncol 2013;8:346-51.

16. Hanahan D, Weinberg RA. Hallmarks of cancer: the next generation. Cell 2011;144:646-74.

17. Wood SL, Pernemalm M, Crosbie PA, et al. The role of the tumor-microenvironment in lung cancer-metastasis and its relationship to potential therapeutic targets. Cancer Treat Rev 2014;40:558-66.

18. Martin TR, Frevert CW. Innate immunity in the lungs. Proc Am Thorac Soc 2005;2:403-11.

19. Schoenhals JE, Seyedin SN, Anderson C, et al. Uncovering the immune tumor microenvironment in non-small cell lung cancer to understand response rates to checkpoint blockade and radiation. Transl Lung Cancer Res 2017;6:148-58.

20. Suzuki T, Chow CW, Downey GP. Role of innate immune cells and their products in lung immunopathology. Int J Biochem Cell Biol 2008;40:1348-61.

21. Ortiz ML, Lu L, Ramachandran I, et al. Myeloid-derived suppressor cells in the development of lung cancer. Cancer Immunol Res 2014;2:50-8.

22. Min L, Mohammad Isa SA, Shuai $W$, et al. Cutting edge: granulocyte-macrophage colony-stimulating factor is the major CD8+ T cell-derived licensing factor for dendritic cell activation. J Immunol 2010;184:4625-9.

23. Wong MT, Ong DE, Lim FS, et al. A High-Dimensional Atlas of Human T Cell Diversity Reveals TissueSpecific Trafficking and Cytokine Signatures. Immunity 2016;45:442-56.

24. Reyes DK, Pienta KJ. The biology and treatment of oligometastatic cancer. Oncotarget 2015;6:8491-524.

25. Pan SH, Chao YC, Hung PF, et al. The ability of LCRMP-1 to promote cancer invasion by enhancing filopodia formation is antagonized by CRMP-1. J Clin Invest 2011;121:3189-205.

26. Tischler V, Pfeifer M, Hausladen S, et al. L1CAM protein expression is associated with poor prognosis in non-small cell lung cancer. Mol Cancer 2011;10:127.

27. Haraguchi M, Okubo T, Miyashita Y, et al. Snail regulates cell-matrix adhesion by regulation of the expression of integrins and basement membrane proteins. J Biol Chem 2008;283:23514-23.

28. Hung JJ, Yang MH, Hsu HS, et al. Prognostic significance of hypoxia-inducible factor-1alpha, TWIST1 and Snail expression in resectable non-small cell lung cancer. Thorax 2009;64:1082-9.

29. Grivennikov SI, Karin M. Inflammation and oncogenesis: a vicious connection. Curr Opin Genet Dev 2010;20:65-71.
30. Wang W, Li Q, Yamada T, et al. Crosstalk to stromal fibroblasts induces resistance of lung cancer to epidermal growth factor receptor tyrosine kinase inhibitors. Clin Cancer Res 2009;15:6630-8.

31. Bar J, Feniger-Barish R, Lukashchuk N, et al. Cancer cells suppress p53 in adjacent fibroblasts. Oncogene 2009;28:933-6.

32. Chatzistamou I, Dioufa N, Trimis G, et al. p21/waf1 and smooth-muscle actin alpha expression in stromal fibroblasts of oral cancers. Anal Cell Pathol (Amst) 2010;33:19-26.

33. Honda E, Yoshida K, Munakata H. Transforming growth factor-beta upregulates the expression of integrin and related proteins in MRC-5 human myofibroblasts. Tohoku J Exp Med 2010;220:319-27.

34. Massague J. TGFbeta in Cancer. Cell 2008;134:215-30.

35. An J, Enomoto A, Weng L, et al. Significance of cancerassociated fibroblasts in the regulation of gene expression in the leading cells of invasive lung cancer. J Cancer Res Clin Oncol 2013;139:379-88.

36. Neaud V, Faouzi S, Guirouilh J, et al. Human hepatic myofibroblasts increase invasiveness of hepatocellular carcinoma cells: evidence for a role of hepatocyte growth factor. Hepatology 1997;26:1458-66.

37. Wald O, Izhar U, Amir G, et al. Interaction between neoplastic cells and cancer-associated fibroblasts through the CXCL12/CXCR4 axis: role in non-small cell lung cancer tumor proliferation. J Thorac Cardiovasc Surg 2011;141:1503-12.

38. Kinoshita T, Ishii G, Hiraoka N, et al. Forkhead box $\mathrm{P} 3$ regulatory $\mathrm{T}$ cells coexisting with cancer associated fibroblasts are correlated with a poor outcome in lung adenocarcinoma. Cancer Sci 2013;104:409-15.

39. Bergfeld SA, DeClerck YA. Bone marrow-derived mesenchymal stem cells and the tumor microenvironment. Cancer Metastasis Rev 2010;29:249-61.

40. De Miguel MP, Fuentes-Julian S, Blazquez-Martinez A, et al. Immunosuppressive properties of mesenchymal stem cells: advances and applications. Curr Mol Med 2012;12:574-91.

41. Noel D, Djouad F, Bouffi C, et al. Multipotent mesenchymal stromal cells and immune tolerance. Leuk Lymphoma 2007;48:1283-9.

42. Liu L, Ge D, Ma L, et al. Interleukin-17 and prostaglandin E2 are involved in formation of an M2 macrophagedominant microenvironment in lung cancer. J Thorac Oncol 2012;7:1091-100.

43. Wang R, Zhang J, Chen S, et al. Tumor-associated 
macrophages provide a suitable microenvironment for non-small lung cancer invasion and progression. Lung Cancer 2011;74:188-96.

44. Wei L, Song XR, Sun JJ, et al. Lysyl oxidase may play a critical role in hypoxia-induced NSCLC cells invasion and migration. Cancer Biother Radiopharm 2012;27:672-7.

45. Gonzalez-Arriaga P, Pascual T, Garcia-Alvarez A, et al. Genetic polymorphisms in MMP 2, 9 and 3 genes modify lung cancer risk and survival. BMC Cancer 2012;12:121.

46. Sauter W, Rosenberger A, Beckmann L, et al. Matrix metalloproteinase 1 (MMP1) is associated with earlyonset lung cancer. Cancer Epidemiol Biomarkers Prev 2008;17:1127-35.

47. Costello CM, McCullagh B, Howell K, et al. A role for the CXCL12 receptor, CXCR7, in the pathogenesis of human pulmonary vascular disease. Eur Respir J 2012;39:1415-24.

48. Hernandez L, Magalhaes MA, Coniglio SJ, et al. Opposing roles of CXCR4 and CXCR7 in breast cancer metastasis. Breast Cancer Res 2011;13:R128.

49. Gridley T. Notch signaling during vascular development. Proc Natl Acad Sci U S A 2001;98:5377-8.

50. Kopp HG, Placke T, Salih HR. Platelet-derived transforming growth factor-beta down-regulates NKG2D thereby inhibiting natural killer cell antitumor reactivity. Cancer Res 2009;69:7775-83.

51. Richter U, Schroder C, Wicklein D, et al. Adhesion of small cell lung cancer cells to E- and P-selectin under physiological flow conditions: implications for metastasis formation. Histochem Cell Biol 2011;135:499-512.

52. St Hill CA. Interactions between endothelial selectins and cancer cells regulate metastasis. Front Biosci (Landmark Ed) 2011;16:3233-51.

53. Kim YN, Koo KH, Sung JY, et al. Anoikis resistance: an essential prerequisite for tumor metastasis. Int J Cell Biol 2012;2012:306879.

54. Kuang BH, Wen XZ, Ding Y, et al. The prognostic value of platelet endothelial cell adhesion molecule- 1 in nonsmall-cell lung cancer patients. Med Oncol 2013;30:536.

55. Galaup A, Cazes A, Le Jan S, et al. Angiopoietin-like 4 prevents metastasis through inhibition of vascular permeability and tumor cell motility and invasiveness. Proc Natl Acad Sci U S A 2006;103:18721-6.

56. Lee BC, Lee TH, Avraham S, et al. Involvement of the chemokine receptor CXCR4 and its ligand stromal cellderived factor 1alpha in breast cancer cell migration through human brain microvascular endothelial cells. Mol Cancer Res 2004;2:327-38.

57. Bauer K, Mierke C, Behrens J. Expression profiling reveals genes associated with transendothelial migration of tumor cells: a functional role for alphavbeta3 integrin. Int J Cancer 2007;121:1910-8.

58. Paget $\mathrm{S}$. The distribution of secondary growths in cancer of the breast. 1889. Cancer Metastasis Rev 1989;8:98-101.

59. Psaila B, Lyden D. The metastatic niche: adapting the foreign soil. Nat Rev Cancer 2009;9:285-93.

60. Valenzuela Alvarez M, Gutierrez LM, Correa A, et al. Metastatic Niches and the Modulatory Contribution of Mesenchymal Stem Cells and Its Exosomes. Int J Mol Sci 2019;20:1946.

61. Quint LE, Tummala S, Brisson LJ, et al. Distribution of distant metastases from newly diagnosed non-small cell lung cancer. Ann Thorac Surg 1996;62:246-50.

62. Belperio JA, Phillips RJ, Burdick MD, et al. The SDF-1/ CXCL 12/CXCR4 biological axis in non-small cell lung cancer metastases. Chest 2004;125:156S.

63. Kijima T, Maulik G, Ma PC, et al. Regulation of cellular proliferation, cytoskeletal function, and signal transduction through CXCR4 and c-Kit in small cell lung cancer cells. Cancer Res 2002;62:6304-11.

64. Improta G, Zupa A, Fillmore H, et al. Protein pathway activation mapping of brain metastasis from lung and breast cancers reveals organ type specific drug target activation. J Proteome Res 2011;10:3089-97.

65. Kim R, Keam B, Kim S, et al. Differences in tumor microenvironments between primary lung tumors and brain metastases in lung cancer patients: therapeutic implications for immune checkpoint inhibitors. BMC Cancer 2019;19:19.

66. Kudo Y, Haymaker C, Zhang J, et al. Suppressed immune microenvironment and repertoire in brain metastases from patients with resected non-small-cell lung cancer. Ann Oncol 2019;30:1521-30.

67. Peng X, Guo W, Ren T, et al. Differential expression of the RANKL/RANK/OPG system is associated with bone metastasis in human non-small cell lung cancer. PLoS One 2013;8:e58361.

68. Peters S, Meylan E. Targeting receptor activator of nuclear factor-kappa B as a new therapy for bone metastasis in nonsmall cell lung cancer. Curr Opin Oncol 2013;25:137-44.

69. Liu L, Chen X, Wang Y, et al. Notch3 is important for TGF-beta-induced epithelial-mesenchymal transition in non-small cell lung cancer bone metastasis by regulating ZEB-1. Cancer Gene Ther 2014;21:364-72.

70. Brodt P, Fallavollita L, Khatib AM, et al. Cooperative regulation of the invasive and metastatic phenotypes by different domains of the type I insulin-like growth factor 
receptor beta subunit. J Biol Chem 2001;276:33608-15.

71. Burnier JV, Wang N, Michel RP, et al. Type IV collageninitiated signals provide survival and growth cues required for liver metastasis. Oncogene 2011;30:3766-83.

72. Yoshimura K, Meckel KF, Laird LS, et al. Integrin alpha2 mediates selective metastasis to the liver. Cancer Res 2009;69:7320-8.

73. Sato S, Hanibuchi M, Kuramoto T, et al. Macrophage stimulating protein promotes liver metastases of small cell lung cancer cells by affecting the organ microenvironment. Clin Exp Metastasis 2013;30:333-44.

74. Castanon E, Soltermann A, Lopez I, et al. The inhibitor of differentiation-1 (Id1) enables lung cancer liver colonization through activation of an EMT program in tumor cells and establishment of the pre-metastatic niche. Cancer Lett 2017;402:43-51.

75. Raynaud CM, Mercier O, Dartevelle P, et al. Expression of chemokine receptor CCR6 as a molecular determinant of adrenal metastatic relapse in patients with primary lung cancer. Clin Lung Cancer 2010;11:187-91.

76. Rotow J, Bivona TG. Understanding and targeting resistance mechanisms in NSCLC. Nat Rev Cancer 2017;17:637-58.

77. Bae SY, Park HJ, Hong JY, et al. Down-regulation of SerpinB2 is associated with gefitinib resistance in nonsmall cell lung cancer and enhances invadopodia-like structure protrusions. Sci Rep 2016;6:32258.

78. Yamauchi M, Yoshino I, Yamaguchi R, et al. N-cadherin expression is a potential survival mechanism of gefitinibresistant lung cancer cells. Am J Cancer Res 2011;1:823-33.

79. Suzuki E, Yamazaki S, Naito T, et al. Secretion of high amounts of hepatocyte growth factor is a characteristic feature of cancer-associated fibroblasts with EGFR-TKI resistance-promoting phenotype: A study of 18 cases of cancer-associated fibroblasts. Pathol Int 2019;69:472-80.

80. Schmid S, Fruh M, Peters S. Targeting MET in EGFR resistance in non-small-cell lung cancer-ready for daily practice? Lancet Oncol 2020;21:320-2.

81. Bronte G, Bravaccini S, Bronte E, et al. Epithelial-tomesenchymal transition in the context of epidermal growth factor receptor inhibition in non-small-cell lung cancer. Biol Rev Camb Philos Soc 2018;93:1735-46.

82. Sequist LV, Waltman BA, Dias-Santagata D, et al. Genotypic and histological evolution of lung cancers acquiring resistance to EGFR inhibitors. Sci Transl Med 2011;3:75ra26.

83. Murakami A, Takahashi F, Nurwidya F, et al. Hypoxia increases gefitinib-resistant lung cancer stem cells through the activation of insulin-like growth factor 1 receptor. PLoS One 2014;9:e86459.

84. Muz B, de la Puente P, Azab F, et al. The role of hypoxia in cancer progression, angiogenesis, metastasis, and resistance to therapy. Hypoxia (Auckl) 2015;3:83-92.

85. Chung FT, Lee KY, Wang CW, et al. Tumor-associated macrophages correlate with response to epidermal growth factor receptor-tyrosine kinase inhibitors in advanced nonsmall cell lung cancer. Int J Cancer 2012;131:E227-35.

86. Matsumoto Y, Sawa K, Fukui M, et al. Impact of tumor microenvironment on the efficacy of epidermal growth factor receptor-tyrosine kinase inhibitors in patients with EGFR-mutant non-small cell lung cancer. Cancer Sci 2019;110:3244-54.

87. Yamada T, Takeuchi S, Nakade J, et al. Paracrine receptor activation by microenvironment triggers bypass survival signals and ALK inhibitor resistance in EML4-ALK lung cancer cells. Clin Cancer Res 2012;18:3592-602.

88. Kogita A, Togashi Y, Hayashi H, et al. Hypoxia induces resistance to ALK inhibitors in the $\mathrm{H} 3122$ non-small cell lung cancer cell line with an ALK rearrangement via epithelial-mesenchymal transition. Int J Oncol 2014;45:1430-6.

89. Passiglia F, Pilotto S, Facchinetti F, et al. Treatment of advanced non-small-cell lung cancer: The 2019 AIOM (Italian Association of Medical Oncology) clinical practice guidelines. Crit Rev Oncol Hematol 2020;146:102858.

90. De Guillebon E, Dardenne A, Saldmann A, et al. Beyond the concept of cold and hot tumors for the development of novel predictive biomarkers and the rational design of immunotherapy combination. Int J Cancer 2020;147:1509-18.

91. Galon J, Bruni D. Approaches to treat immune hot, altered and cold tumours with combination immunotherapies. Nat Rev Drug Discov 2019;18:197-218.

92. Botticelli A, Cerbelli B, Lionetto L, et al. Can IDO activity predict primary resistance to anti-PD-1 treatment in NSCLC? J Transl Med 2018;16:219.

93. Brochez L, Chevolet I, Kruse V. The rationale of indoleamine 2,3-dioxygenase inhibition for cancer therapy. Eur J Cancer 2017;76:167-82.

94. Fridman WH, Pages F, Sautes-Fridman C, et al. The immune contexture in human tumours: impact on clinical outcome. Nat Rev Cancer 2012;12:298-306.

95. Gopalakrishnan V, Spencer CN, Nezi L, et al. Gut microbiome modulates response to anti-PD-1 immunotherapy in melanoma patients. Science 2018;359:97-103. 
96. Sade-Feldman M, Yizhak K, Bjorgaard SL, et al. Defining T Cell States Associated with Response to Checkpoint Immunotherapy in Melanoma. Cell 2018;175:998-1013.e20.

97. Simoni Y, Becht E, Fehlings M, et al. Bystander CD8(+) $T$ cells are abundant and phenotypically distinct in human tumour infiltrates. Nature 2018;557:575-9.

98. Robertson J, Salm M, Dangl M. Adoptive cell therapy with tumour-infiltrating lymphocytes: the emerging importance of clonal neoantigen targets for next-generation products in non-small cell lung cancer. Immuno-Oncology Technology 2019;3:1-7.

99. Law AMK, Valdes-Mora F, Gallego-Ortega D. MyeloidDerived Suppressor Cells as a Therapeutic Target for Cancer. Cells 2020;9:561.

100. Bertelli G, Trovato R, Ugel S, et al. Characterization of Myeloid-derived Suppressor Cells in a Patient With Lung Adenocarcinoma Undergoing Durvalumab Treatment: A Case Report. Clin Lung Cancer 2019;20:e514-6.

101.Jin J, Yang L, Liu D, et al. Association of the neutrophil to lymphocyte ratio and clinical outcomes in patients with lung cancer receiving immunotherapy: a meta-analysis. BMJ Open 2020;10:e035031.

102. Mezquita L, Auclin E, Ferrara R, et al. Association of the Lung Immune Prognostic Index With Immune Checkpoint Inhibitor Outcomes in Patients With Advanced Non-Small Cell Lung Cancer. JAMA Oncol 2018;4:351-7.

103. Wong-Rolle A, Wei HK, Zhao C, et al. Unexpected guests in the tumor microenvironment: microbiome in cancer. Protein Cell 2020. doi: 10.1007/s13238-020-00813-8.

104.Fessler J, Matson V, Gajewski TF. Exploring the emerging role of the microbiome in cancer immunotherapy. J Immunother Cancer 2019;7:108.

105.Wong-Rolle A, Wei HK, Zhao C, et al. Unexpected guests in the tumor microenvironment: microbiome in cancer. Protein Cell 2021;12:426-35.

106. Routy B, Le Chatelier E, Derosa L, et al. Gut microbiome influences efficacy of PD-1-based immunotherapy against epithelial tumors. Science 2018;359:91-7.

107.Pienta KJ, Robertson BA, Coffey DS, et al. The cancer diaspora: Metastasis beyond the seed and soil hypothesis. Clin Cancer Res 2013;19:5849-55.

108. Kim C, Hoang CD, Kesarwala AH, et al. Role of Local Ablative Therapy in Patients with Oligometastatic and Oligoprogressive Non-Small Cell Lung Cancer. J Thorac Oncol 2017;12:179-93.

109. Taniguchi K, Okami J, Kodama K, et al. Intratumor heterogeneity of epidermal growth factor receptor mutations in lung cancer and its correlation to the response to gefitinib. Cancer Sci 2008;99:929-35.

110.Schmid K, Oehl N, Wrba F, et al. EGFR/KRAS/ BRAF mutations in primary lung adenocarcinomas and corresponding locoregional lymph node metastases. Clin Cancer Res 2009;15:4554-60.

111.Park S, Holmes-Tisch AJ, Cho EY, et al. Discordance of molecular biomarkers associated with epidermal growth factor receptor pathway between primary tumors and lymph node metastasis in non-small cell lung cancer. J Thorac Oncol 2009;4:809-15.

112.Chen ZY, Zhong WZ, Zhang XC, et al. EGFR mutation heterogeneity and the mixed response to EGFR tyrosine kinase inhibitors of lung adenocarcinomas. Oncologist 2012;17:978-85.

113. Turajlic S, Xu H, Litchfield K, et al. Tracking Cancer Evolution Reveals Constrained Routes to Metastases: TRACERx Renal. Cell 2018;173:581-94.e12.

114.Pitroda SP, Khodarev NN, Huang L, et al. Integrated molecular subtyping defines a curable oligometastatic state in colorectal liver metastasis. Nat Commun 2018;9:1793.

115.Khodarev NN, Pitroda SP, Weichselbaum RR. microRNAs and oligometastasis. Aging (Albany NY) 2015;7:146-7.

116. Ottaiano A, Circelli L, Lombardi A, et al. Genetic trajectory and immune microenvironment of lungspecific oligometastatic colorectal cancer. Cell Death Dis 2020;11:275.
Cite this article as: Belluomini L, Dodi A, Caldart A, Kadrija D, Sposito M, Casali M, Sartori G, Ferrara MG, Avancini A, Bria E, Menis J, Milella M, Pilotto S. A narrative review on tumor microenvironment in oligometastatic and oligoprogressive non-small cell lung cancer: a lot remains to be done. Transl Lung Cancer Res 2021;10(7):3369-3384. doi: 10.21037/tlcr-20-1134 